\title{
Coefficient Estimates for Subclasses of Bi-Univalent Functions
}

\author{
Waggas Galib Atshan Rajaa Ali Hiress \\ Department of Mathematics, College of Computer Science and \\ Information Technology, University of AI- Qadisiyah, Diwaniya-Iraq \\ waggas . galib@qu.edu.iq waggashnd@gmail.com
}

Available online : $\quad 5 / 8 / 2018$

DOI: $10.29304 / j q c m .2018 .10 .3 .401$

\begin{abstract}
In the present paper, we introduce two new subclasses of the class $\sum$ consisting of analytic and bi-univalent functions in the open unit disk $U$. Also, we obtain the estimates on the TaylorMaclurin coefficients $\left|a_{2}\right|$ and $\left|a_{3}\right|$ for functions in these subclasses. We obtain new special cases for our results.
\end{abstract}

Keywords : Analytic function, Univalent function , Bi-univalent function , Coefficient estimates .

Mathematics Subject Classification : 30C45. 


\section{Introduction}

Let $\mathcal{H}$ be the class of the functions of the form :

$f(z)=z+\sum_{j=2}^{\infty} a_{j} z^{j}, \quad(z \in U)$,

which are analytic in the open unit disk $U=\{z: z \in$ $\mathbb{C}$ and $|z|<1\}$. Also, let $S$ denoted the class of all functions in $\mathcal{H}$ which are univalent and normalized by the conditions $f(0)=0=f^{\prime}(0)-1$ in $U[1]$. It is well known that every univalent function $f$ has inverse $f^{-1}$ satisfying:

$f^{-1}(f(z))=z(z \in U)$,

and

$f\left(f^{-1}(w)\right)=w\left(|w|<r_{0}(f) ; r_{0}(f) \geq \frac{1}{4}\right)$,

where

$f^{-1}(w)=g(w)=w-a_{2} w^{2}+\left(2 a_{2}^{2}-a_{3}\right) w^{3}-$

$\left(5 a_{2}^{3}-5 a_{2} a_{3}+a_{4}\right) w^{4}+\cdots$.

A function $f \in \mathcal{H}$ is said to be bi-univalent in $U$ if both $f(z)$ and $f^{-1}(z)$ are univalent in $U$. Let $\sum$ denote the class of bi-univalent functions defined in the unit disk $U$ given by (1.1). For a brief history and interesting example in the class $\sum$ (see [2]). However , the familiar Koebe function is not bi-univalent. The class $\sum$ of bi-univalent functions was first inverstigated by Lewin [3] and it was shown that $\left|a_{2}\right|<1.51$. Brannan and Clunie [4] improved Lewin's result and conjectured that $\left|a_{2}\right| \leq \sqrt{2}$. Later, Netanyahu [5], showed that if $f \in \sum$, then $\max \left|a_{2}\right|=\frac{4}{3}$.

Recently, Srivastava et al. [6], Frasin and Aouf [7], BansaL and Sokol [8] and Srivastava and BansaL [2] are also introduced and investigated the various subclasses of bi- univalent functions and obtained bounds for the initial coefficients $\left|a_{2}\right|$ and $\left|a_{3}\right|$.

The coefficient estimate problem involving the bound of $\left|a_{n}\right|(n \in N \backslash\{1,2\} ; N=\{1,2,3, \ldots\})$ for each $f \in \sum$ given by (1.1) is still an open problem.
The object of this work is to find estimates on the Taylor -Maclaurin coefficients $\left|a_{2}\right|$ and $\left|a_{3}\right|$ for functions in this subclasses $S_{\Sigma}(\tau, \gamma, \delta ; \alpha)$ and $S_{\Sigma}(\tau, \gamma, \delta ; \beta)$ of the functions class $\sum$. Several related classes are also considered and connections to earlier known results are made.

In order to prove in our main results, we require the following lemma.

Lemma 1.1.[1] If $h \in p$ the $\left|c_{k}\right| \leq 2$ for each $k$, where $p$ is the family of all functions $h$ analytic in $U$ for which $\operatorname{Re}(h(z))>0$ $h(z)=1+c_{1} z+c_{2} z^{2}+c_{3} z^{3}+\cdots$. for $z \in U$

\section{Coefficient Bounds for Function class} $S_{\Sigma}(\tau, \gamma, \delta ; \alpha)$

To prove our main results, we need to introduce the following definition .

Definition 2. 1. A function $f(z)$ given by (1.1) is said to be in the class $S_{\Sigma}(\tau, \gamma, \delta ; \alpha)(\tau \in \mathbb{C} \backslash\{0\}, 0 \leq \gamma \leq$ $1,0 \leq \delta<1,0<\alpha \leq$ ) if the following conditions are satisfied :

$f \in$

$\sum, \mid \arg \left(1+\frac{1}{\tau}\left[\frac{z\left(f^{\prime}(z)+\gamma z f^{\prime \prime}(z)\right)}{\gamma z\left(f^{\prime}(z)+\delta z f^{\prime \prime}(z)\right)+(1-\gamma)\left(\delta z f^{\prime}(z)+(1-\delta) f(z)\right)}-\right.\right.$

1])|

$<\frac{\alpha \pi}{2} \quad(z \in U)$

and

$g \in \sum,\left|\arg \left(1+\frac{1}{\tau}\left[\frac{w\left(g^{\prime}(w)+\gamma w g^{\prime \prime}(w)\right)}{w \gamma\left(g^{\prime}(w)+\delta g^{\prime \prime}(w)\right)+(1-\gamma)\left(\delta w g^{\prime}(w)+(1-\delta) g(w)\right)}-1\right]\right)\right|$ $<\frac{\alpha \pi}{2} \quad(w \in U)$

where the function $g(w)$ is given by (1.2).

Theorem 2.2. Let $f(z)$ given by (1.1) be in the class $S_{\Sigma}(\tau, \gamma, \delta ; \alpha)(\tau \in \mathbb{C} \backslash\{0\}, 0 \leq \gamma \leq 1,0 \leq \delta<$ $1,0<\alpha \leq 1)$. Then $\left|a_{2}\right| \leq$

$\frac{2 \alpha|\tau|}{\sqrt{\left|2 \tau \alpha\left((2-2 \delta+4 \gamma-4 \delta \gamma)-\left(1+2 \gamma+\gamma^{2}-2 \delta^{2} \gamma-\delta^{2}-\delta^{2} \gamma^{2}\right)\right)+(1-\alpha)(1-\delta+\gamma-\delta \gamma)^{2}\right|}}$ 
and

$\left|a_{3}\right| \leq \frac{2|\tau| \alpha}{|(2-2 \delta+4 \gamma-4 \delta \gamma)|}+\frac{4|\tau|^{2} \alpha^{2}}{(1-\delta+\gamma-\delta \gamma)^{2}}$.

Proof: Let $f(z) \in S_{\Sigma}(\tau, \gamma, \delta ; \alpha)$.Then

$1+\frac{1}{\tau}\left[\frac{z\left(f^{\prime}(z)+\gamma z f^{\prime \prime}(z)\right)}{\gamma z\left(f^{\prime}(z)+\delta z f^{\prime \prime}(z)\right)+(1-\gamma)\left(\delta z f^{\prime}(z)+(1-\delta) f(z)\right)}-1\right]=$

$[r(z)]^{\alpha}$

and

$1+\frac{1}{\tau}\left[\frac{w\left(g^{\prime}(w)+\gamma w g^{\prime \prime}(w)\right)}{w \gamma\left(g^{\prime}(w)+\delta g^{\prime \prime}(w)\right)+(1-\gamma)\left(\delta w g^{\prime}(w)+(1-\delta) g(w)\right)}-1\right]=$ $[h(w)]^{\alpha}$.

Where $r(z)$ and $h(w)$ are in $p$ and have the following series representations :

$r(z)=1+r_{1} z+r_{2} z^{2}+r_{3} z^{3}+\cdots$

and

$h(w)=1+h_{1} w+h_{2} w^{2}+h_{3} w^{3}+\cdots$.

Since

$1+\frac{1}{\tau}\left[\frac{z\left(f^{\prime}(z)+\gamma z f^{\prime \prime}(z)\right)}{\gamma z\left(f^{\prime}(z)+\delta z f^{\prime \prime}(z)\right)+(1-\gamma)\left(\delta z f^{\prime}(z)+(1-\delta) f(z)\right)}-1\right]=$ $1+\frac{1}{\tau}(1-\delta+\gamma-\delta \gamma) a_{2} z+\frac{1}{\tau}\left((2-2 \delta+4 \gamma-4 \delta \gamma) a_{3}\right.$ $-\left(1+2 \gamma+\gamma^{2}-2 \delta^{2} \gamma-\delta^{2}-\delta^{2} \gamma^{2}\right) a_{2}^{2} z^{2}+$

$\cdots$,

and

$1+\frac{1}{\tau}\left[\frac{w\left(g^{\prime}(w)+\gamma w g^{\prime \prime}(w)\right)}{w \gamma\left(g^{\prime}(w)+\delta g^{\prime \prime}(w)\right)+(1-\gamma)\left(\delta w g^{\prime}(w)+(1-\delta) g(w)\right)}-\right.$

$1]=1-\frac{1}{\tau}(1-\delta+\gamma-\delta \gamma) a_{2} w+\frac{1}{\tau}((2-2 \delta+4 \gamma-$

$4 \delta \gamma)$

$\left(2 a_{2}^{2}-a_{3}\right)-\left(1+2 \gamma+\gamma^{2}-2 \delta^{2} \gamma-\delta^{2}-\delta^{2} \gamma^{2}\right) a_{2}^{2} w^{2}+\cdots$.

Now , equating the coefficients in (2.5) and (2.6), we get

$\frac{1}{\tau}(1-\delta+\gamma-\delta \gamma) a_{2}=\alpha r_{1}$,

$\frac{1}{\tau}\left((2-2 \delta+4 \gamma-4 \delta \gamma) a_{3}-\left(1+2 \gamma+\gamma^{2}-2 \delta^{2} \gamma-\delta^{2}\right.\right.$

$\left.\left.-\delta^{2} \gamma^{2}\right) a_{2}^{2}\right)=\alpha r_{2}+r_{1}^{2} \frac{\alpha(\alpha-1)}{2}$

$-\frac{1}{\tau}(1-\delta+\gamma-\delta \gamma) a_{2}=\alpha h_{1}$

and $\frac{1}{\tau}\left((2-2 \delta+4 \gamma-4 \delta \gamma)\left(2 a_{2}^{2}-a_{3}\right)-\left(1+2 \gamma+\gamma^{2}-2 \delta^{2} \gamma\right.\right.$

$\left.-\delta^{2}-\delta^{2} \gamma^{2}\right) a_{2}^{2}=\alpha h_{2}+h_{1}^{2} \frac{\alpha(\alpha-1)}{2}$.

From (2.11) and (2.13), we find

$r_{1}=-h_{1}$

and

$\frac{2}{\tau^{2}}(1-\delta+\gamma-\delta \gamma)^{2} a_{2}^{2}=\alpha^{2}\left(r_{1}^{2}+h_{1}^{2}\right)$.

Also, from (2.12), (2.14) and (2.16), we find that

$\frac{2}{\tau}\left((2-2 \delta+4 \gamma-4 \delta \gamma) a_{2}^{2}-\right.$

$\left.\left(1+2 \gamma+\gamma^{2}-2 \delta^{2} \gamma-\delta^{2}-\delta^{2} \gamma^{2}\right) a_{2}^{2}\right)=\alpha\left(r_{2}+h_{2}\right)+$ $\frac{\alpha(\alpha-1)}{2}\left(r_{1}^{2}+h_{1}^{2}\right)=\alpha\left(r_{2}+h_{2}\right)+\frac{(\alpha-1)}{\alpha \tau^{2}}(1-\delta+\gamma-$

$\delta \gamma)^{2} a_{2}^{2}$

Therefore, we obtain

$a_{2}^{2}=$

$\frac{\alpha^{2} \tau^{2}\left(r_{2}+h_{2}\right)}{2 \alpha \tau\left((2-2 \delta+4 \gamma-4 \delta \gamma)-\left(1+2 \gamma+\gamma^{2}-2 \delta^{2} \gamma-\delta^{2}-\delta^{2} \gamma^{2}\right)\right)+(1-\alpha)(1-\delta+\gamma-\delta \gamma)^{2}}$.

Applying Lemma (1.1) for the coefficients $r_{2}$ and $h_{2}$, we readily

get

$\left|a_{2}\right| \leq$

$\frac{2 \alpha|\tau|}{\sqrt{\left|2 \tau \alpha\left((2-2 \delta+4 \gamma-4 \delta \gamma)-\left(1+2 \gamma+\gamma^{2}-2 \delta^{2} \gamma-\delta^{2}-\delta^{2} \gamma^{2}\right)\right)+(1-\alpha)(1-\delta+\gamma-\delta \gamma)^{2}\right|}}$

The last inequality gives the desired estimate on $\left|a_{2}\right|$ given in (2.3).

Next, in order to find the bound on $\left|a_{3}\right|$, by subtracting (2.12) and (2.14), we get

$\frac{1}{\tau}(2-2 \delta+4 \gamma-4 \delta \gamma)\left(2 a_{3}-2 a_{2}^{2}\right)=\left(\alpha r_{2}+r_{1}^{2} \frac{\alpha(\alpha-1)}{2}\right)$

$-\left(\alpha h_{2}+h_{1}^{2} \frac{\alpha(\alpha-1)}{2}\right)$.

It follows from $(2.15),(2.16)$ and (2.18), that

$$
\begin{aligned}
\left.a_{3}=\frac{\alpha \tau\left(r_{2}-h_{2}\right)}{2(2-2 \delta+}+4 \gamma-4 \delta \gamma\right) & \\
& +\frac{\alpha^{2} \tau^{2}\left(r_{1}^{2}-h_{1}^{2}\right)}{2(1-\delta+\gamma-\delta \gamma)^{2}} .
\end{aligned}
$$

Applying Lemma (1.1) once again for the coefficients $r_{1}, r_{2}, h_{1}$ and $h_{2}$, we immediately 


$$
\begin{aligned}
\left.\left|a_{3}\right| \leq \mid \frac{2 \alpha|\tau|}{(2-2 \delta}+4 \gamma-4 \delta \gamma\right) & \\
& +\frac{4|\tau|^{2} \alpha^{2}}{(1-\delta+\gamma-\delta \gamma)^{2}} .
\end{aligned}
$$

This complete the proof of Theorem (2.2).

\section{Coefficient Bounds for Function class $S_{\Sigma}(\tau, \gamma, \delta ; \beta)$}

To prove our main results, we need to introduce the following definition .

Definition 3. 1. A function $f(z)$ given by (1.1) is said to be in the class $S_{\Sigma}(\tau, \gamma, \delta ; \beta)(\tau \in \mathbb{C} \backslash\{0\}, 0 \leq \gamma \leq$ $1,0 \leq \delta<1,0 \leq \beta<1$ ) if the following conditions are satisfied :

$f \in$

$\sum, \operatorname{Re}\left(1+\frac{1}{\tau}\left[\frac{z\left(f^{\prime}(z)+\gamma z f^{\prime \prime}(z)\right)}{\gamma z\left(f^{\prime}(z)+\delta z f^{\prime \prime}(z)\right)+(1-\gamma)\left(\delta z f^{\prime}(z)+(1-\delta) f(z)\right)}-\right.\right.$

1])

$>\beta \quad(z \in U)$

and

$g \in$

$\sum, \operatorname{Re}\left(1+\frac{1}{\tau}\left[\frac{w\left(g^{\prime}(w)+\gamma w g^{\prime \prime}(w)\right)}{w \gamma\left(g^{\prime}(w)+\delta g^{\prime \prime}(w)\right)+(1-\gamma)\left(\delta w g^{\prime}(w)+(1-\delta) g(w)\right)}-\right.\right.$

1]) $>\beta \quad(w \in U)$,

where the function $g(w)$ is given by (1.2).

Theorem 3.2. Let $f(z)$ given by (1.1) be in the class $S_{\Sigma}(\tau, \gamma, \delta ; \beta)(\tau \in \mathbb{C} \backslash\{0\}, 0 \leq \gamma \leq 1,0 \leq \delta<1,0 \leq$ $\beta<1$ ). Then

$\left|a_{2}\right|=\sqrt{\frac{2|\tau|(1-\beta)}{\left|\left(1+2 \gamma-\gamma^{2}-2 \delta-4 \delta \gamma+2 \delta^{2} \gamma+\delta^{2}+\delta^{2} \gamma^{2}\right)\right|}}$

and

$\left|a_{3}\right| \leq \frac{|\tau|(1-\beta)}{|1-\delta+2 \gamma-2 \delta \gamma|}+\frac{4|\tau|^{2}(1-\beta)^{2}}{\left|(1-\delta+\gamma-\delta \gamma)^{2}\right|}$.

Proof : Let $f(z) \in S_{\Sigma}(\tau, \gamma, \delta ; \beta)$. Then

$$
\begin{gathered}
1+\frac{1}{\tau}\left[\frac{z\left(f^{\prime}(z)+\gamma z f^{\prime \prime}(z)\right)}{\gamma z\left(f^{\prime}(z)+\delta z f^{\prime \prime}(z)\right)+(1-\gamma)\left(\delta z f^{\prime}(z)+(1-\delta) f(z)\right)}-1\right] \\
=\beta+(1-\beta) r(z)
\end{gathered}
$$

and

$$
\begin{aligned}
& 1+\frac{1}{\tau}\left[\frac{w\left(g^{\prime}(w)+\gamma w g^{\prime \prime}(w)\right)}{w \gamma\left(g^{\prime}(w)+\delta g^{\prime \prime}(w)\right)+(1-\gamma)\left(\delta w g^{\prime}(w)+(1-\delta) g(w)\right)}-1\right] \\
& =\beta+(1-\beta) h(w),
\end{aligned}
$$

where $g(w)=f^{-1}(w), r(z)$ and $h(w)$ have form (2.7)and (2.8), respectively.

Now, equating the coefficients in (3.5) and (3.6), we get

$\frac{1}{\tau}(1-\delta+\gamma-\delta \gamma) a_{2}=(1-\beta) r_{1}$,

$\frac{1}{\tau}\left((2-2 \delta+4 \gamma-4 \delta \gamma) a_{3}-\left(1+2 \gamma+\gamma^{2}-2 \delta^{2} \gamma-\right.\right.$

$\left.\left.\delta^{2}-\delta^{2} \gamma^{2}\right) a_{2}^{2}\right)=(1-\beta) r_{2}$,

$-\frac{1}{\tau}(1-\delta+\gamma-\delta \gamma) a_{2}=(1-\beta) h_{1}$,

and

$\frac{1}{\tau}\left((2-2 \delta+4 \gamma-4 \delta \gamma)\left(2 a_{2}^{2}-a_{3}\right)-(1+2 \gamma+\right.$

$\left.\left.\gamma^{2}-2 \delta^{2} \gamma-\delta^{2}-\delta^{2} \gamma^{2}\right) a_{2}^{2}\right)=(1-\beta) h_{2}$.

From (3.7) and (3.9), we obtain

$r_{1}=-h_{1}$

and

$$
\begin{aligned}
\frac{2}{\tau^{2}}(1-\delta+\gamma- & \delta \gamma)^{2} a_{2}^{2} \\
& =(1-\beta)^{2}\left(r_{1}^{2}+h_{1}^{2}\right)
\end{aligned}
$$

Also , from (3.8) and (3.10), we have $\frac{2}{\tau}\left((2-2 \delta+4 \gamma-4 \delta \gamma)-\left(1+2 \gamma+\gamma^{2}-2 \delta^{2} \gamma-\delta^{2}\right.\right.$

$$
\left.\left.-\delta^{2} \gamma^{2}\right)\right) a_{2}^{2}
$$

$=(1-\beta)\left(r_{2}+h_{2}\right)$.

Therefore, we get

$a_{2}^{2}=\frac{\tau(1-\beta)\left(r_{2}+h_{2}\right)}{2\left((2-2 \delta+4 \gamma-4 \delta \gamma)-\left(1+2 \gamma+\gamma^{2}-2 \delta^{2} \gamma-\delta^{2}-\delta^{2} \gamma^{2}\right)\right)}$.

Applying Lemma (1.1) for coefficients $r_{2}$ and $h_{2}$, we obtain

$\left|a_{2}\right| \leq \sqrt{\frac{2|\tau|(1-\beta)}{\left|\left(1+2 \gamma-\gamma^{2}-2 \delta-4 \delta \gamma+2 \delta^{2} \gamma+\delta^{2}+\delta^{2} \gamma^{2}\right)\right|}}$.

This gives the bound on $\left|a_{2}\right|$ as asserted in (3.3).

Next in order to find the bound on $\left|a_{3}\right|$, by subtracting (3.8) and (3.10), we thus get 
$\frac{1}{\tau}(2-2 \delta+4 \gamma-4 \delta \gamma)\left(2 a_{3}-2 a_{2}^{2}\right)=(1-\beta)\left(r_{2}-h_{2}\right)$

or, equivalently,

$a_{3}=\frac{\tau(1-\beta)\left(r_{2}-h_{2}\right)}{4(1-\delta+2 \gamma-2 \delta \gamma)}+a_{2}^{2}$

It follows from (3.12) and (3.17), that

$a_{3}=\frac{\tau(1-\beta)\left(r_{2}-h_{2}\right)}{4(1-\delta+2 \gamma-2 \delta \gamma)}+\frac{\tau^{2}(1-\beta)^{2}\left(r_{1}^{2}+h_{1}^{2}\right)}{2(1-\delta+\gamma-\delta \gamma)^{2}}$.

Applying Lemma (1.1) once again for the coefficients $r_{1}, r_{2}, h_{1}$ and $h_{2}$, we obtain

$\left|a_{3}\right| \leq \frac{|\tau|(1-\beta)}{|(1-\delta+2 \gamma-2 \delta \gamma)|}+\frac{4|\tau|^{2}(1-\beta)^{2}}{\left|(1-\delta+\gamma-\delta \gamma)^{2}\right|}$.

This completes the prove of Theorem (3.2).

\section{Corollaries and Consequence}

This section is devoted to the presentation of some special cases of the main results .

These results are given in the form of corollaries :

If we set $\tau=1$ and $\delta=0$ in Theorems (2.2) and (3.2), then, we get following results due to Keerthi and Raja [9] :

Corollary 4.1. Let $f(z)$ given by (1.1) be in the class $\mathcal{B}_{\Sigma}(\gamma ; \alpha)(0 \leq \gamma \leq 1,0<\alpha \leq 1)$. Then

$\left|a_{2}\right| \leq \frac{2 \alpha}{\sqrt{\left|4 \alpha(1+2 \gamma)+(1-3 \alpha)(\gamma+1)^{2}\right|}}$

and

$\left|a_{3}\right| \leq \frac{4 \alpha^{2}}{(1+\gamma)^{2}}+\frac{\alpha}{1+2 \gamma}$.

Corollary 4.2. Let $f(z)$ given by $(1.1)$ be in the class $\mathcal{B}_{\Sigma}(\gamma ; \beta)(0 \leq \gamma \leq 1,0 \leq \beta<1)$. Then

$\left|a_{2}\right| \leq \sqrt{\frac{2(1-\beta)}{\left|1+2 \gamma-\gamma^{2}\right|}}$

and

$\left|a_{3}\right| \leq \frac{4(1-\beta)^{2}}{(1+\gamma)^{2}}+\frac{1-\beta}{1+2 \gamma}$.

The classes $\mathcal{B}_{\Sigma}(\gamma ; \alpha)$ and $\mathcal{B}_{\Sigma}(\gamma ; \beta) \quad$ are respectively defined as follows:
Definition 4.3. A function $f(z)$ given by (1.1) is said to be in the class $\mathcal{B}_{\Sigma}(\gamma ; \alpha)(0 \leq \gamma \leq 1,0<\alpha \leq 1)$ if the following conditions are satisfied:

$f \in \sum,\left|\arg \left(\frac{z\left(f^{\prime}(z)+\gamma z f^{\prime \prime}(z)\right)}{(1-\gamma) f(z)+\gamma z f^{\prime}(z)}\right)\right|<\frac{\alpha \pi}{2} \quad(z \in U)$

and

$g \in \sum,\left|\arg \left(\frac{w\left(g^{\prime}(w)+\gamma w g^{\prime \prime}(w)\right)}{(1-\gamma) g(w)+\gamma w g^{\prime}(w)}\right)\right|<\frac{\alpha \pi}{2}, \quad(w \in U)$ where the function $g(w)$ is given by (1.2) .

Definition 4. 4. A function $f(z)$ given by (1.1) is said to be in the class

$\mathcal{B}_{\Sigma}(\beta ; \gamma)(0 \leq \gamma \leq 1,0 \leq \beta<1)$ if the following conditions are satisfied:

$f \in \sum, \operatorname{Re}\left(\frac{z\left(z f^{\prime}(z)+\gamma z f^{\prime \prime}(z)\right)}{(1-\gamma) f(z)+\gamma z f^{\prime}(z)}\right)>\beta \quad(z \in U)$

and

$g \in \sum, \operatorname{Re}\left(\frac{w\left(g^{\prime}(w)+\gamma w g^{\prime \prime}(w)\right)}{(1-\gamma) g(w)+\gamma z g^{\prime}(w)}\right)$

$$
>\beta, \quad(w \in U)
$$

where the function $g(w)$ is given by (1.2).

If we set $\tau=1$ and $\gamma=0$ in Theorems (2.2) and (3.2), then the classes $S_{\Sigma}(\tau, \gamma, \delta ; \alpha)$ and $S_{\Sigma}(\tau, \gamma, \delta ; \beta)$ reduce to the classes $\mathcal{G}_{\Sigma}(\delta ; \alpha)$ and $\mathcal{G}_{\Sigma}(\delta ; \beta) \quad$ investigated by Murugusundaramoorthy et al. [10] ,which are defined as follows :

Definition 4.5. $A$ function $f(z)$ given by (1.1) is said to be in the class $\mathcal{G}_{\Sigma}(\delta ; \alpha)(0<\alpha \leq 1,0 \leq \delta<1)$ if the following conditions are satisfied:

$$
\begin{array}{r}
f \in \sum,\left|\arg \left(\frac{z f^{\prime}(z)}{(1-\delta) f(z)+\delta f^{\prime}(z)}\right)\right| \\
<\frac{\alpha \pi}{2} \quad(z \in U)
\end{array}
$$

and

$$
\begin{array}{r}
g \in \sum,\left|\arg \left(\frac{w g^{\prime}(w)}{(1-\delta) g(w)+\delta g^{\prime}(w)}\right)\right| \\
<\frac{\alpha \pi}{2}, \quad(w \in U)
\end{array}
$$

where the function $g(w)$ is given by (1.2). 
Definition 4.6. A function $f(z)$ given by (1.1) is said to be in the class $\mathcal{G}_{\Sigma}(\delta ; \beta)(0 \leq \beta<1,0 \leq \delta<1)$ if the following conditions are satisfied:

$f \in \sum, \operatorname{Re}\left(\frac{z f^{\prime}(z)}{(1-\delta) f(z)+\delta f^{\prime}(z)}\right)>\beta \quad(z \in U)$

where and

$$
\begin{gathered}
g \in \sum, \operatorname{Re}\left(\frac{w g^{\prime}(w)}{(1-\delta) g(w)+\delta g^{\prime}(w)}\right) \\
>\beta, \quad(w \in U)
\end{gathered}
$$

the function $g(w)$ is is given by (1.2).

In this case .Theorems (2.2) and (3.2) reduce to the following:

Corollary 4.7. Let $f(z)$ given by (1.1)be in the class $\mathcal{G}_{\Sigma}(\delta ; \alpha)(0<\alpha \leq 1,0 \leq \delta<1)$.Then

$\left|a_{2}\right| \leq \frac{2 \alpha}{(1-\delta) \sqrt{(\alpha+1)}}$

and

$\left|a_{3}\right| \leq \frac{4 \alpha^{2}}{(1-\delta)^{2}}+\frac{\alpha}{1-\delta}$.

Corollary 4. 8. Let $f(z)$ given by $(1.1)$ be in the class $\mathcal{G}_{\Sigma}(\delta ; \alpha)(0 \leq \beta<1,0 \leq \delta<1)$.Then

$\left|a_{2}\right| \leq \frac{\sqrt{2(1-\beta)}}{(1-\delta)}$

and

$\left|a_{2}\right| \leq \frac{4(1-\beta)^{2}}{(1-\delta)^{2}}+\frac{1-\beta}{(1-\delta)}$

Letting $\tau=1$ and $\gamma=1$ in Theorems (2.2) and (3.2) gives the following corollaries:

Corollary 4.9. Let $f(z)$ given by (1.1) be in the class $\mathcal{D}_{\Sigma}(\delta ; \alpha)(0<\alpha \leq 1,0 \leq \delta<1)$.Then

$\left|a_{2}\right|$

$\leq \frac{2 \alpha}{\sqrt{\left.\mid 2 \alpha(6-6 \delta)-\left(4-4 \delta^{2}\right)\right)+(1-\alpha)(2-2 \delta)^{2} \mid}}$ and

$\left|a_{3}\right| \leq \frac{\alpha}{3(1-\delta)}+\frac{\alpha^{2}}{(1-\delta)^{2}}$.
Corollary 4.10. Let $f(z)$ given by (1.1) be in the class $\mathcal{D}_{\Sigma}(\delta ; \beta)(0 \leq \beta<1,0 \leq \delta<1)$.Then

$\left|a_{2}\right| \leq \sqrt{\frac{(1-\beta)}{\left|2-3 \delta+2 \delta^{2}\right|}}$

and

$\left|a_{3}\right| \leq \frac{(1-\beta)}{|3-3 \delta|}+\frac{(1-\beta)^{2}}{(1-\delta)^{2}}$.

The classes $\mathcal{D}_{\Sigma}(\delta ; \alpha)$ and $\mathcal{D}_{\Sigma}(\tau, \delta ; \beta)$ are given explicitly in the next definitions .

Definition 4.11. A function $f(z)$ given by (1.1) is said to be in the class $\mathcal{D}_{\Sigma}(\delta ; \alpha)(0<\alpha \leq 1,0 \leq \delta<$ 1) if the following conditions are satisfied :

$f \in \sum,\left|\arg \left(\frac{f^{\prime}(z)+z f^{\prime \prime}(z)}{f^{\prime}(z)+\delta z f^{\prime \prime}(z)}\right)\right|<\frac{\alpha \pi}{2} \quad(z \in U)$

and

$$
\begin{array}{r}
g \in \sum,\left|\arg \left(\frac{g^{\prime}(w)+w g^{\prime \prime}(w)}{g^{\prime}(w)+\delta w g^{\prime \prime}(w)}\right)\right| \\
<\frac{\alpha \pi}{2}, \quad(w \in U)
\end{array}
$$

where the function $g(w)$ is given by (1.2).

Definition 4.12. A function $f(z)$ given by (1.1) is said to be in the class $\mathcal{D}_{\Sigma}(\delta ; \beta)(0 \leq \beta<1,0 \leq \delta<$ 1) if the following conditions are satisfied:

$f \in \sum, \operatorname{Re}\left(\frac{f^{\prime}(z)+z f^{\prime \prime}(z)}{f^{\prime}(z)+\delta z f^{\prime \prime}(z)}\right)>\beta \quad(z \in U)$

and

$g \in \sum, \operatorname{Re}\left(\frac{g^{\prime}(w)+w g^{\prime \prime}(w)}{g^{\prime}(w)+\delta g^{\prime \prime} g(w)}\right)>\beta, \quad(w \in U)$

where the function $g(w)$ is given by (1.2). 


\section{References}

[1] P. Duren ,Univalent Functions , Grandlehrender Mathematischen Wissenscaften,259, Springer, New York ,(1983).

[2] H. M. Srivastava and D. Bansal , Coefficient estimates for a subclass of analytic and bi- univalent functions, J .Egypt. Math. Soc.,1-4, (2014).

[3] M. Lewin ,On a coefficient problem for biunivalent functions ,Proc. Am. Math. Soc.,18, 63-68, (1967).

[4] D.A. Brannan ,J. Clunie Aspects of contemporary complex Analysis ,Academic press ,New York London,(1980).

[5] E. Netanyahau, The minimal distance of the image boundary from the origin and the second coefficient of a univalent function in $|z|<1$, Arch. Rational Mech. Anal., 32(2),100-112, (1969).
[6] H. M. Srivastava ,A. K. Mishra and P. Gochhayat ,Certain subclasses of analytic and biunivalent functions ,appl. Math. Lett., 23,1188-1192, (2010).

[7] B.A. Frasin , M. K. Aouf ,New subclasses of biunivalent functions ,Appl. Math. Lett., 24 (9),1569-

1573, (2011).

[8] D. BansaL ,J. Sokol, Coefficient bound for a new class of analytic and bi-univalent functions, J. Fract . Clac. Appl., 5(1), 122-128,(2014).

[9] B. S. Keerthi, B. Raja, Coefficient inequality for certain new subclasses of analytic bi- univalent functions, Theoretical Mathematics and Applications, 31(1),1-10, (2013).

[10] G. Murugusundaramoorthy , N. Magesh , V. Prameela , Coefficient bounds for certain subclasses of bi-univalent function, Abstr. Appl. Anal., 2013(Article ID 573017),1-3),(2013).

\section{مخمنات المعامل لاصناف جزئبه من الدوال الثنائية التكاقؤ وقاص غالب عطشان رجاء علي هريس \\ قسم الرياضيات / كلية علوم الحاسوب وتكنولوجيا المعلومات / جامعة القادسية}

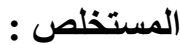

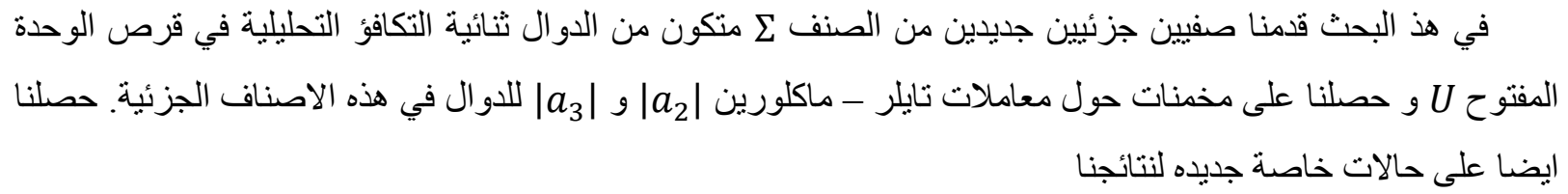

